\title{
Tarifkonflikte aus spieltheoretischer Perspektive
}

Im Augenblick hat man das Gefühl, es wird fast ständig gestreikt. Der Eindruck trügt. Deutschland ist im internationalen Vergleich nach wie vor ganz am unteren Ende der Statistik. Die gestiegene Wahrnehmung liegt wohl daran, dass in letzter Zeit vor allem der Verkehr sowie der öffentliche Dienst betroffen waren, Bereiche, die alle angehen. Dennoch ist die Frage berechtigt, ob man Tarifkonflikte generell nicht anders lösen könnte. Ein Instrument, das helfen kann diese Frage zu beantworten, ist die Spieltheorie. Sie analysiert Entscheidungssituationen, in denen mehrere Subjekte Entscheidungen, die sich gegenseitig beeinflussen, treffen. Die Subjekte können Personen oder auch Institutionen sein, also etwa ein Arbeitgeberverband oder eine Gewerkschaft. Die Komplexität eines strategischen Entscheidungsproblems entsteht daraus, dass die optimale Entscheidung des einen von der Entscheidung des anderen abhängt und umgekehrt. Die Entscheidung der Arbeitgeber, ein bestimmtes Angebot zu unterbreiten, kann die Entscheidung der Gegenseite beeinflussen zu streiken oder weiter zu verhandeln.

Die Spieltheorie fordert dabei nicht, dass sich die Beteiligten egoistisch verhalten. Die Rationalitätsannahme unterstellt, dass sich die Beteiligten an ihren eigenen Zielen orientieren, und versucht dann, das Verhalten der Beteiligten so gut wie möglich zu prognostizieren, um letztlich für jeden Beteiligten eine individuell optimale Entscheidung zu finden. Die Beteiligten dürfen dabei egoistisch oder aber auch altruistisch sein. Die Spieltheorie zeigt jedoch ein zentrales Problem derart strategischer Entscheidungen auf: Individuelle Rationalität und kollektive Rationalität stimmen häufig nicht überein. Wenn jeder nur an sich denkt, kommt nicht notwendigerweise ein insgesamt sinnvolles Ergebnis zustande.

Interessanterweise sollte dieses Problem aus einer theoretischen Perspektive gerade bei Verhandlungen nicht auftreten. Die Grundlagen der Verhandlungstheorie gehen unter anderem auf den Ökonomen Ariel Rubinstein zurück. Er zeigt, dass sich rationale Verhandlungspartner grundsätzlich ohne Verursachung von Kosten einigen sollten. Diese Überlegung lässt sich anhand der Tarifverhandlungen einfach darstellen: Starten wir dazu mit der abschließenden Einigung. Die Tarifparteien haben sich bisher noch immer geeinigt, möglicherweise erst nach langen und kostspieligen Verhandlungen. Könnten die Vertragsparteien durch eine gute Analyse dieses abschließende Ergebnis vorhersehen, so könnten sich beide Parteien dadurch besserstellen, dass sie sich vor dem Streik auf genau dasselbe Ergebnis einigen. Beide Seiten könnten dadurch Kosten sparen, ohne das Endergebnis zu verändern. Die Gewerkschaften dürfen dabei nicht vollständig auf das Instrument Streik und die Bereitschaft zu streiken verzichten. Die Drohung allein sollte aber genügen, die Gegenseite dazu zu bewegen, das akzeptable Angebot zu unterbreiten.

Weshalb kommt es dann zu Streiks, zum Scheitern von Klimaverhandlungen oder zu Kriegen? Sind die Beteiligten zu schlecht vorbereitet? Schätzen sie die Situation falsch ein? Auch das kann ein zentraler Punkt sein. Aber selbst bei spieltheoretisch perfekt geschulten Verhandlungspartnern gibt es Gründe, weshalb Verhandlungen scheitern oder Kosten entstehen, die alle Beteiligten gern vermeiden möchten. Ein wichtiger Grund ist unzureichende Information über die Verhandlungspartner. Man kann davon ausgehen, dass in Abhängigkeit von den Rahmenbedingungen einer Branche unterschiedliche Verhandlungsergebnisse infrage kommen. Eine boomende Branche kann ohne Weiteres deutlich höhere Lohnabschlüsse akzeptieren, eine

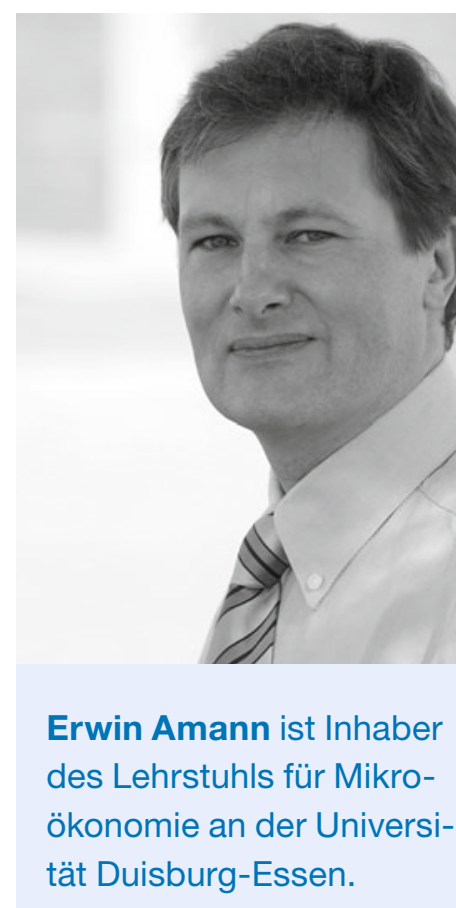


Branche unter Druck hingegen kann so hohe Abschlüsse nicht verkraften. Ist dies auch den Gewerkschaften bewusst, so werden sich die jeweils realistischen Lohnabschlüsse durchsetzen. Ist die Gewerkschaft hingegen über die Lage der Branche nicht informiert, so haben die Arbeitgeber immer einen Anreiz, die Situation schlechter darzustellen, als sie tatsächlich ist. Damit die Arbeitgeber dazu motiviert werden, freiwillig die Lage korrekt darzustellen, kann ein Streik dienen. Die Kosten des Streiks sind für Unternehmen in einem boomenden Markt naturgemäß höher. Sie laufen Gefahr, lukrative Aufträge zu verlieren. Ist die Branche hingegen unter Druck, schrecken Streiks weit weniger, da die Unternehmen bei zu hohen Lohnabschlüssen langfristig Schwierigkeiten zu überleben hätten. Lassen sich also hohe Lohnabschlüsse durchsetzen, werden die Unternehmen relativ rasch auf die Forderungen der Gewerkschaften eingehen, um Streiks abzuwenden. Branchen, die nicht ausgelastet sind, werden Streiks hingegen hinnehmen.

Treffen diese Argumente zu, so sollten in Branchen mit Streiks vergleichsweise niedrige Abschlüsse zu erwarten sein. Bessere Abschlüsse erzielen die Gewerkschaften in jenen Branchen, in denen Streiks vermieden werden. Arbeitnehmer, die in den Streik eintreten, sollten sich also tendenziell mit niedrigen Abschlüssen anfreunden. Umgekehrt sind Unternehmen gut beraten, den Arbeitnehmern ihre Situation transparent darzustellen. Je geringer das Informationsdefizit, desto höher die Chance, auch in schlechten Zeiten niedrige Lohnabschlüsse ohne Streik durchzusetzen. Dies ist naturgemäß in der Privatwirtschaft leichter. Die angespannte Haushaltslage von Kommunen oder Ländern überzeugt Gewerkschaften möglicherweise wenig. Vielleicht ist dies ein Grund, weshalb in Deutschland überproportional häufig im öffentlichen Dienst gestreikt wird. Grundsätzlich ist aber auch das Scheitern von Tarifverhandlungen aus anderen als den genannten Ursachen denkbar. Vielleicht sind sich die Gewerkschaften bewusst, dass hohe Abschlüsse nicht durchzusetzen sind, stehen aber unter Druck, den eigenen Mitgliedern nicht einfach geringe Abschlüsse präsentieren zu können. Der Streik ist somit nur dazu da überzeugend darzulegen, dass man alles dafür getan hat, ein sehr schlechtes Angebot wenigstens in einigen Punkten zu verbessern. Hätten die Arbeitgeber von Anfang an das letztlich akzeptierte Angebot unterbreitet, könnten die Gewerkschaften keine Erfolge präsentieren und würden Mitglieder verlieren.

In diesem Fall lassen sich Streiks möglicherweise gar nicht vermeiden. Sie verursachen zwar gesellschaftliche Kosten, erzeugen jedoch einen nicht auf den ersten Blick sichtbaren und vor allem nicht nach außen kommunizierbaren Nutzen, der im Verhandlungsprozess dennoch relevant ist. Die Gewerkschaft erreicht eine stärkere Legitimation unter den Arbeitnehmern, die sie vertritt, und erhöht entsprechend ihre Mitgliederzahl. Dies könnte in den aktuellen Verhandlungen zwischen der Gewerkschaft Deutscher Lokomotivführer (GDL) und der Deutschen Bahn AG (DB AG) der Fall sein. Die GDL muss nicht nur gute Abschlüsse verhandeln, das könnte sie sicherlich auch ohne Streik, sie muss sich auch in Konkurrenz zur Eisenbahn- und Verkehrsgewerkschaft (EVG) profilieren. Hinzu kommt in diesem Fall, dass das geplante Gesetz zur Tarifeinheit die Macht der kleineren Gewerkschaft GDL im Vergleich zu der größeren EVG erheblich einschränkt. Der Fakt, dass die DB AG eine Schlichtung sucht, die GDL dieser jedoch nicht zustimmt, lässt diese Vermutung als sehr plausibel erscheinen.

Wie aber könnte die DB AG in dieser Situation handeln, um einen Streik abzuwenden? Dreier-Verhandlungen sind auch aus theoretischer Sicht nicht unbedingt er-

Erwin Amann

Universität Duisburg-Essen erwin.amann@uni-due.de strebenswert. Die EVG wiederum hat kein Interesse, Entgegenkommen zu zeigen. Eine friedliche Lösung erscheint in diesem Fall auch aus spieltheoretischer Sicht fast ausgeschlossen. 